\title{
THE UAV SIMULATION COMPLEX FOR OPERATOR TRAINING
}

\author{
Oleksandr Volkov, Mykola Komar, Kateryna Synytsya and Dmytro Volosheniuk \\ International Research and Training Center for Information Technologies and Systems \\ 40, Glushkova ave. Kyiv 03187, Ukraine
}

\begin{abstract}
UAV operators training programs vary depending on the category of the UAV, mission types, requirements to the certification and available platform. The essential part of the UAV operator training is the use of the simulations to facilitate mastering of the piloting skills and operation of the vehicle equipment. Due to the complexity of the simulations, it is desirable to use respective hardware and software complex for a range of training programs. Common topics have been identified based on the analysis of typical training programs, UAV missions and operator functions. The paper outlines the structure of the simulation complex and the framework of the program including simulation-based training and theoretical part which could be supported in a blended mode.
\end{abstract}

\section{KEYWORDS}

Unmanned Aerial Vehicle, Operator Training, UAV Pilot, Flight Simulation

\section{INTRODUCTION}

The term unmanned aerial vehicle (UAV) denotes a wide class of the platforms which either function in an autonomous mode or are remotely operated by a person or a ground team. The UAVs are successfully used in various situations and for various purposes, including civilian and military applications. The primary use of the UAVs was related to missions considered as "dirty, dull, or dangerous" (Elias, B., 2012), such as those related to a hostile environment, situations in which the human health is put on risk, or requiring long-term monitoring which does not require immediate actions. These missions include among others homeland and border security, disaster response, hurricane monitoring, traffic monitoring, application of pesticides. Recent civilian applications extended this list significantly by suggesting research data collection, geospatial imaging, wildlife or natural resources inspection and a variety of trial applications for learning purposes (Shakhatreh, H.et. al, 2018; Crutsinger, G., 2016.). Due to the wide range of potential applications it is important to ensure proper training of UAV operators providing them with the necessary knowledge and skills for the dynamically evolving area.

In Ukraine, UAV services are provided by some specialized organizations only. So far, the UAV operator training has been addressed mostly in security context due to the high cost of the related equipment and lack of the established training requirements. However, due to keen interest in AI, robotics and software engineering advances in the country, the need for efficient training of UAV operators is expected to be growing. The idea behind this research is that basic training could be organized using as a core a flight simulation complex initially developed for academic research of control algorithms. Thus, the purpose of this study is to investigate whether and how this simulation complex could be adapted for training, considering existing requirements and approaches to UAV pilot and operator training. 


\section{UAV OPERATOR TRAINING PROGRAMS}

Expansion of civil and military applications of UAV has led to the appearance of various training programs. These programs are not standardized (Bennett, W., et al, 2016), they are different in volume and objectives and thus difficult to compare. There are several reasons for this variety. First, there are different requirements to the pilot qualification depending on the category of the UAV. Second, many training programs are linked to the specific platform producer, thus focusing on specific hardware, interfaces, and protocols. Third, the programs depend on the potential class of missions, as well as specific team organization ranging from a short course to a special training for pilot license holders (Szabolcsi, R., 2016).

As we intended creating a training environment appropriate for both military and civilian operator training, the next step was to determine respective UAV categories, most typical missions, and operator functions to start with. It has been anticipated that operator training could be similar for certain mission types that share many common operator functions.

In the absence of generally accepted classification of the UAV, several approaches have been studied, starting with (Korchenko, A., 2013) which considers 16 features describing construction, components, and behavior of the vehicle, as well as its category, size, and typical missions. In a more targeted description (Qi, C., 2018), six categories of the UAV are proposed based on the following characteristics: maximum take-off weight, altitude, radius of operation, endurance, i.e. maximum flight time, and typical use. For current modeling purposes, we follow the NASA classification which is limited to three categories based on UAV weight and speed as most representative characteristics. Although the computer modeling has been done for all three types, e.g. High Altitude Long Endurance (HALE), Medium Range (MR), and Mini plane types UAVs that have different aerodynamic characteristics and are used for different missions, the training program development is focused on MR and Mini UAVs.

Our analysis of the UAV missions and operator function taxonomies (Nehme, 2007; Pavlas, 2009) resulted in the selection of the missions related to intelligence, reconnaissance, and surveillance as those applicable in both civilian and military context. Common operator functions for all of them are monitoring the status of the UAV, resource allocation and scheduling, path planning, position or target tracking, monitoring sensors and analyzing sensor data. The last one is specific for mission and sensors. Skills for operators that actually pilot the small UAV also include take-off and landing, cruise, navigation, technical inspection, and reporting.

Most UAV pilot and operator training programs offer some volume of general learning content that explains the basics of aerodynamics, meteorology, electronics and other disciplines. This content is not specific to the vehicle or mission and provides necessary background for operation of the flying vehicle. Another important component of the training program is related to safety issues and regulations, local/global legislation for flying vehicles, risk factors and operation in abnormal situations. Table 1 illustrates how certain topics could be addressed by using the simulation complex, classroom training with access to the real samples of UAV and devices, and distance learning content and tests offered via the LMS Moodle.

Table 1. A general structure of the operator training program

\begin{tabular}{lll}
\hline Module & Example of the topic & Learning Environment \\
\hline General knowledge & Meteorology, navigation & Classroom, Moodle \\
UAV & Components & Hands-on, Moodle \\
Safety \& Regulations & Safety procedures & Moodle \\
Equipment & Sensors & Hands-on, Moodle \\
Reporting & Data analysis & Moodle \\
Piloting & Take-off and landing & Simulation complex \\
Mission simulation & Traffic control & Simulation complex \\
\hline
\end{tabular}

\section{UAV SIMULATION COMPLEX}

Initially, the UAV simulation complex has been created to support research and development of control algorithms for UAV, in particular, in cases when non-linear model cannot be easily approximated by a linear one. Currently, it contains a collection of plane-type models and software enabling simulation of the UAV behavior in an auto-pilot mode and as a reaction to the manual control. Besides watching the computer 
simulation of the UAV behavior, the user can observe the behavior of the movable parts, control elements, and engine workload of the real sample of the UAV which is connected to the simulation complex.

The core of the UAV simulation complex contains the main computer with UAV manual flight control device and three displays intended for visualizing the behavior of the UAV, its control dashboard and other simulation-related information (fig.1). The computational environment consists of five main modules implementing dynamic behavior of the UAV, databases management, processing of the simulation parameters, mission and flight information, processing of the on-board control, and visualization of the UAV flight.

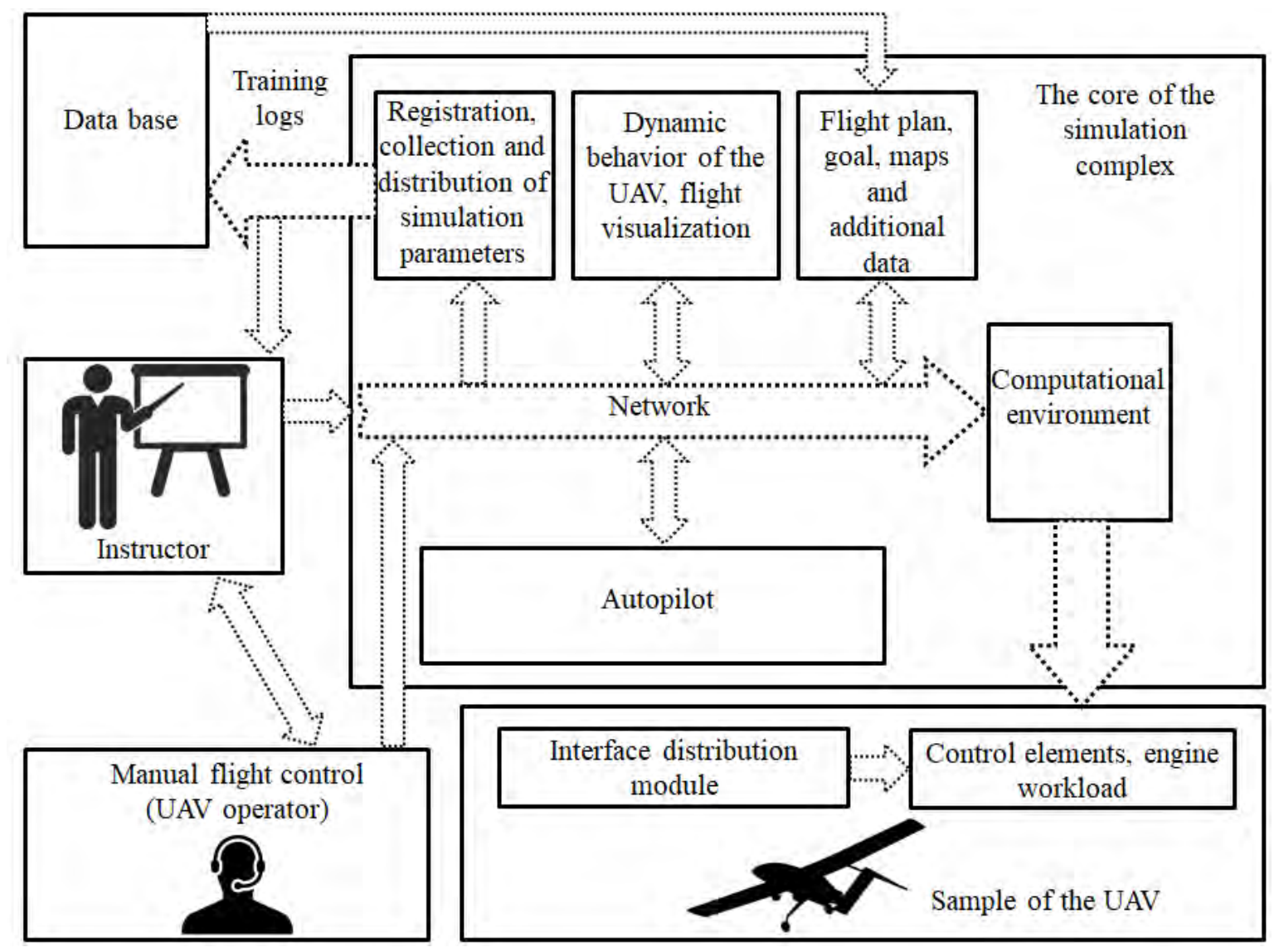

Figure 1. A general scheme of the UAV simulation complex

There are several options to implement training using the simulation complex. First, a student can be given a mission case to accomplish individually using a manual control of the UAV. The parameters of his/her performance are registered and thus the behavior can be reproduced by the simulator for trainer's evaluation and group discussion. For an immediate feedback, a trainer may observe the student's behavior and information available for him, such as dashboard indicators, maps, sensors (not only in the lab but also remotely) and intercept the UAV control or communicate with the student to correct his mistake. There is also an option to specify thresholds for students' deviation from the behavior simulated for a particular case so that they will be warned that remediation is necessary.

To support training of the piloting skills for the Mini UAV located in the lab, a set of tasks (mission cases and maps) has been prepared. Related theoretical part will contain the modules related to the UAV-specific knowledge, such as UAV components (airframe, engine, navigation and control system, on-board payload, launch and landing systems), ground control systems (control panels, communication equipment, and sensors), systems and procedures for UAV pre-flight preparation, take-off and landing, maintenance and repair. 


\section{CONCLUSION}

The proposed approach illustrates the benefits of the existing simulation complex use for the UAV operator training. A common core of skills and knowledge of the UAV revealed in the study allows for suggesting a structure of the blended training program as a combination of simulations, classroom lessons and learning content deliverable via the LMS used by the organization. The proposed modular training program advancing from the basic skills and knowledge related to the UAV operation and arranged around the existing simulation complex may be adapted and extended to address the specific needs. The construction of the simulation complex allows for extensions (equipment, sensors, and models). At the moment, an additional workstation may be connected to simulate the behavior of a foe UAV (target) or partner UAV operating within the same mission. Therefore, the UAV simulation complex may support UAV pilot training for both military and civilian missions and is extendable to the team training.

\section{ACKNOWLEDGEMENT}

This research was partly supported by the grant of the National academy of sciences of Ukraine for young researchers groups and laboratories.

\section{REFERENCES}

Al-Tahir, R., 2015. Integrating UAV into geomatics curriculum. The International Archives of Photogrammetry, Remote Sensing and Spatial Information Sciences, Vol. 40, No.1, pp.387-390.

Bennett, W., et al, 2016. Training issues for remotely piloted aircraft systems from a human systems integration perspective. In Cooke, N., et al (eds.) Remotely Piloted Aircraft Systems: A Human Systems Inte gration Perspective, pp.163-176. John Wiley \& Sons, Ltd.

Crutsinger, G.M., et al, 2016. The future of UAVs in ecology: an insider perspective from the Silicon Valley drone industry. Journal of Unmanned Aerial Systems, Vol. 4, pp. 161-168.

Elias, B., 2012. Pilotless drones: Background and considerations for Congress regarding unmanned aircraft operations in the national airspace system. CRS Report to Congress. Congressional Research Service.

Korchenko A. G. and Illyash O. S., 2013. The generalized classification of Unmanned Air Vehicles, Proceedings of the 2nd IEEE Conference on Actual Problems of Unmanned Air Vehicles Developments (APUAVD), Kiev, pp. 28-34.

Nehme, C.E., et al, 2007, June. An operator function taxonomy for unmanned aerial vehicle missions. Proceedings of the 12th International Command and Control Research and Technology Symposium. Washington, DC, pp. 1-9.

Pavlas, D. Et al., 2009, October. Enhancing unmanned aerial system training: A taxonomy of knowledge, skills, attitudes, and methods. Proceedings of the Human Fa ctors and Ergonomics Soci ety A nnual Me eting, Vol. 53, No. 26, pp. 1903-1907. Sage CA: Los Angeles, CA: SAGE Publications.

Qi, S., et al, 2018. Unmanned Aircraft System Pilot/Operator Qualification Requirements and Training Study. Proceedings of the $M$ ATECWeb of Co nferences. Wuhan, China. Vol. 179, 03006. EDP Sciences. DOI: $10.1051 /$ matecconf/201817903006.

Shakhatreh, H.et. al, 2018. Unmanned aerial vehicles: A survey on civil applications and key research challenges. IEEE Access. Vol.7. DOI: 7.10.1109/ACCESS.2019.2909530.

Szabolcsi, R., 2016, June. Beyond Training Minimums - A New Concept of the UAV Operator Training Program. Proceedings of the International con ference K nowledge-Based Organization. Sibiu, Romania. Vol. 22, No. 3, pp. 560-566. De Gruyter Open. 